\title{
Canadian Urological Association guideline: Management of small renal masses - Summary of recommendations
}

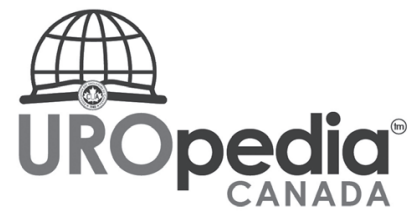

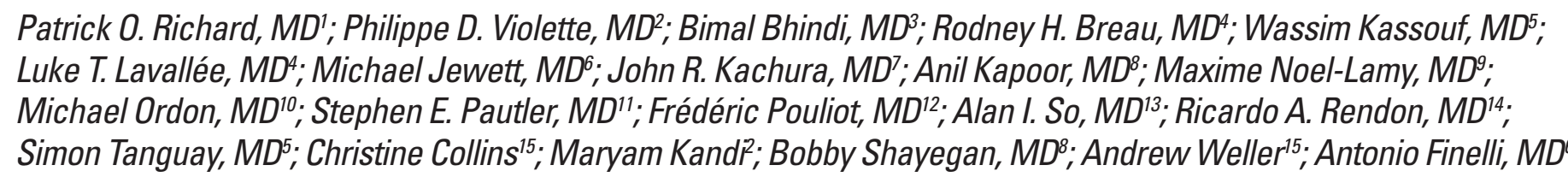

'Department of Surgery, Division of Urology, Centre Hospitalier Universitaire de Sherbrooke, Sherbrooke, QC, Canada; ${ }^{2}$ Departments of Health Research Methods Evidence and Impact (HEI) and Surgery, McMaster University, Hamilton, ON, Canada; ${ }^{3}$ Southern Alberta Institute of Urology, University of Calgary, Calgary, AB, Canada; ${ }^{4}$ Department of Surgery, Division of Urology, University of Ottawa, Ottawa, ON, Canada; ${ }^{5}$ Department of Surgery, Division of Urology, McGill University Health Centre, Montreal, QC, Canada; ${ }^{6}$ Department of Surgical Oncology, Division of Urology, Princess Margaret Hospital, Toronto, ON, Canada; ${ }^{7}$ Joint Department of Medical Imaging, University Health Network, University of Toronto, Toronto, ON, Canada; ${ }^{8}$ McMaster Institute of Urology, St. Joseph Healthcare, Hamilton, ON, Canada; ${ }^{9}$ Department of Medical Imaging, Division of Interventional Radiology, Centre Hospitalier Universitaire de Sherbrooke, Sherbrooke, QC, Canada; ${ }^{10}$ Department of Surgery, Division of Urology, St. Michael's Hospital, Toronto, ON, Canada; "'Department of Surgery, Division of Urology, Schulich School of Medicine and Dentistry, Western University, London, ON, Canada; ${ }^{12 D e p a r t m e n t ~ o f ~ S u r g e r y, ~ D i v i s i o n ~ o f ~}$ Urology, Centre Hospitalier Universitaire de Québec, Quebec, QC, Canada; ${ }^{13}$ Division of Urology, British Columbia Cancer Care, Vancouver, BC, Canada; ${ }^{14}$ Department of Surgery, Division of Urology, Capital Health - QEII, Halifax, NS, Canada; ${ }^{15}$ Kidney Cancer Canada, Mississauga, ON, Canada

\section{Reviewers: Andrea Kokorovic, $M D^{\dagger}$; Jay Nayak, $M D^{\ddagger}$}

†Centre Hospitalier de I'Université de Montréal, Montreal, QC, Canada; ”Department of Surgery, Rady Faculty of Health Sciences, University of Manitoba, Winnipeg, MB, Canada

Cite as: Richard PO, Violette PD, Bhindi B, et al. Canadian Urological Association guideline: Management of small renal masses - Summary of recommendations. Can Urol Assoc J 2022;16(2):24-5. http://dx.doi.org/10.5489/cuaj.7760

Below are recommendations from the CUA on the management of small renal masses (SRM). The full-text guideline, found at cuaj.ca and cua.org, includes a detailed description of methodology, as well as the summary of evidence in support of these recommendations.

1. Patients diagnosed with SRM should undergo routine laboratory investigations, including at a minimum a serum creatinine and glomerular filtration rate (Clinical principle).

2. Patients with SRM incidentally discovered on routine imaging should be investigated with a multiphasic, contrast-enhanced abdominal computed tomography (CT) or magnetic resonance imaging (MRI) scan (Clinical principle).

3. For patients with suspected renal malignancy, a baseline chest X-ray is suggested to assess for pulmonary metastases (Conditional recommendation, low certainty in evidence of effects).

4. Patients with SRM and pre-existing renal dysfunction in whom a radical nephrectomy is being considered, may be offered renal scintigraphy when the result may alter their management (Clinical principle).

5. Patients with SRM should be offered a renal mass biopsy when the result of the biopsy may alter their management
(Adopted from Kidney Cancer Research Network of Canada [KCRNC] consensus on the role of renal mass biopsy in the management of kidney cancer; expert opinion).

6. Patients with features suspicious of hereditary renal cell carcinoma (RCC) should be offered genetic counselling (Adopted from CUA guideline on genetic screening for hereditary RCC; expert opinion).

7. For patients with SRM suspicious for renal malignancy AND significant comorbidities and/or limited life expectancy, observation (or watchful waiting) is recommended as the preferred strategy for patients (Strong recommendation, high certainty in evidence of effects).

8. For patients with a suspected renal malignancy measuring $<2 \mathrm{~cm}$ in diameter, active surveillance is suggested as the preferred strategy, given their slow growth rate and low probability of aggressive histology (Conditional recommendation, moderate certainty in evidence of effects).

9. For patients with a suspected renal malignancy measuring $2-4 \mathrm{~cm}$ in diameter, active surveillance and definitive treatment (partial nephrectomy or percutaneous thermal ablation) are suggested as management options (Conditional recommendation, low certainty in evidence of effects).

10. For patients with a suspected renal malignancy, the choice of treatment should be personalized using a shared decision-making approach, after proper counselling and while taking into account tumor characteristics, patient factors, and patient preferences and values (Expert opinion). 
11. For patients with a suspected renal malignancy who prefer management by upfront definitive treatment, surgery or percutaneous thermal ablation are suggested (Conditional recommendation, low certainty in evidence of effects).

12. Patients with a suspected renal malignancy who prefer management by upfront definitive treatment should be informed of the higher uncertainty surrounding the data on the efficacy and harms of percutaneous thermal ablation treatment compared to surgery (Expert opinion).

13. Patients with a suspected renal malignancy who opt to be treated by percutaneous thermal ablation should have a renal mass biopsy performed prior to, or at the time of thermal ablation (Adopted from KCRNC consensus on the role of renal mass biopsy in the management of kidney cancer; expert consensus)

14. For patients with suspected malignant SRM undergoing surgery, partial nephrectomy is recommended over radical nephrectomy (Strong recommendation, moderate certainty in evidence of effects).

15. For patients with suspected renal malignancy undergoing partial nephrectomy, a minimally invasive approach (robotic-assisted or conventional laparoscopy) is suggested over an open approach when technically feasible and oncologically safe (Conditional recommendation, moderate certainty in evidence of effects).

16. For patients with suspected renal malignancy undergoing radical nephrectomy, a conventional laparoscopic approach is recommended over open or robotic-assisted approaches (Strong recommendation, moderate certainty in evidence of effects).

17. For patients undergoing percutaneous thermal ablation for a suspected renal malignancy, cryoablation and radio-frequency ablation are both suggested as options for management, as they yield similar oncological outcomes and adverse events (Conditional recommendation, moderate certainty in evidence of effects).

18. Patients under active surveillance should be monitored until the oncological risk increases, they select intervention, or the benefits of treatment outweigh the competing risks. The factors that define oncological risk are not completely elucidated but the most well-accepted factors are: growth of tumor to $>4 \mathrm{~cm}$, consecutive growth rate $>0.5 \mathrm{~cm} /$ year, progression to metastases, and patient's choice (Clinical principle).

19. Patients with suspected tumor growth on ultrasound imaging should undergo cross-sectional imaging to confirm growth prior to intervention (Expert opinion).

20. For patients with suspected renal malignancy who opted to be managed by active surveillance, routine abdominal ultrasound (assuming good visualization and good agreement in size measurements between ultrasound and cross-sectional imaging) is suggested until definitive treatments are no longer considered (i.e., watchful waiting) (Conditional recommendation, low certainty in evidence of effects).

21. For patients with suspected renal malignancy who opted to be managed by active surveillance, chest $\mathrm{X}$-ray imaging is suggested until definitive treatments are no longer considered (i.e., watchful waiting) (Conditional recommendation, low certainty in evidence of effects).

22. The panel was unable to achieve a consensus as to the frequency of abdominal imaging, which varied from at least once every 3-6 months for the first year and then once every 6-12 months if the lesion remains stable. The same can be said regarding the frequency of chest imaging, which varied from for-cause to once a year (Expert opinion).

23. Patients with RCC who have undergone definitive treatment should be followed with routine chest and abdominal imaging to rule out recurrence or progression to metastasis (Adopted from CUA guideline for followup of patients after treatment of non-metastatic RCC; expert opinion).

24. Patients with an estimated glomerular filtration rate (eGFR) $<45 \mathrm{ml} / \mathrm{min} / 1.73 \mathrm{~m}^{2}$ or with progressive chronic kidney disease following definitive treatment should be considered for a referral to a nephrologist (or their general practitioner), especially if associated with proteinuria (Adopted from CUA guideline for followup of patients after treatment of non-metastatic RCC; conditional recommendation, low certainty in evidence of effects).

Competing interests: Dr. Richard has been an advisory board member for Bayer, Janssen, and Sanofi; and a speakers' bureau member for Abbvie, Amgen, Astellas, Ferring, and Janssen. Dr. Bhindi has been an advisory board member for Bayer and Janssen; and has received speaker honoraria from Merck. Dr. Breau has been an advisory board member for Ferring (bladder cancer). Dr. Kassouf has been an advisory board member for EMD Serono and Pfizer; has received grants and/or honoraria from Abbvie, Astellas, BMS, Ferring, Janssen, Merck, Roche, and Sesen Bio; and has participated in clinical trials supported by Astra Zeneca, BMS, Janssen, Pfizer, Roche, Sesen Bio, and Theralase. Dr. Lavallée has participated in advisory boards for Abbvie, Bayer, Ferring, Sanofi, and Tersera; and has received an unrestricted research grant from Sanofi. Dr. Jewett has been an advisory board member for and received payment from Sesen Bio and Theralase Technologies Ltd. Dr. Kachura participated in the multicenter OPTIMA trial for liver cancer ablation supported by Celsion Inc. Dr. Pouliot has been an advisory board member for Astellas, Bayer, Esai, Janssen, Merck, Sanofi, and Tersera; holds investments in Allogene Therapeutics; and has participated in clinical trials supported by Lantheus, Merck, and Progenics. Dr. So has been an advisory board member for Abbvie, Amgen, Bayer, Ferring, Janssen, Merck, and Tersera. Dr. Rendon has been an advisory board and speakers' bureau member for and has received honoraria from Abbvie, Amgen, Astellas, AstraZeneca, Bayer, Ferring, Jansen, and Sanofi. Dr. Tanguay has participated in advisory boards for BMS, Janssen, Knight Therapeutics, Merck, and Roche; and has participated in clinical trials supported by AstraZeneca and Roche. Dr. Shayegan has been an advisory board member for Astellas, Bayer, and Janssen; and has received a research grant from Janssen. Dr. Nayak has been an advisory board member for Merck and Sanofi; and a speakers' bureau member for Astellas, Sanofi, and Tersera. The remaining authors do not report any competing personal or financial interests related to this work.

Correspondence: Dr. Patrick 0. Richard, Division of Urology, Department of Surgery, Centre Hospitalier Universitaire de Sherbrooke, Sherbrooke, QC, Canada; patrick.richard@usherbrooke.ca 